\title{
Pharmacotherapy is an effective treatment option for generalised anxiety disorder
}

Mitte K, Noack P, Steil R, et al. A meta-analytic review of the efficacy of drug treatment in generalized anxiety disorder. J Clin Psychopharmacol 2005;25:141-50.

\section{Q How effective are drugs for the treatment of generalised anxiety disorder?}

METHODS

-

Design: Systematic review and meta-analysis.

Data sources: MEDLINE, PsycLIT (search date May 2002), plus hand search of secondary sources, internet searches, and personal contact with researchers and pharmaceutical companies.

(1) Study selection and analysis: Inclusion criteria: English or German publications of placebo controlled studies of pharmacotherapy for generalised anxiety disorder (DSM or ICD diagnosis, or exact description of disorder), with a minimum of 14 days' treatment, and study size $\geqslant 4$ people. Studies in people refractory to prior pharmacotherapy were excluded. Effect sizes were calculated using Hedges' $\mathrm{g}$ and random effects analysis.

F(2)

Outcomes: Symptoms of depression and anxiety.

\section{MAIN RESULTS}

Forty eight studies met inclusion criteria. Pharmacotherapy reduced symptoms of anxiety more than placebo (effect size $=0.48,95 \% \mathrm{CI}$ 0.32 to 0.64 ). Limiting analysis to studies which used the Hamilton Rating Scale for Anxiety to measure outcomes, or those which included only marketed drugs, did not affect the results significantly. Multiple regression analyses found no significant effect of drug doses, use of a placebo run-in or not, use of different diagnostic tools or dropout rates on analysis of efficacy. There were no significant differences between drug classes (benzodiazepines or azapirones) in effect on anxiety or depression symptoms $(\mathrm{p}>0.05$ for both comparisons). Significantly more people treated with azapirones dropped out than with benzodiazepines $(30.7 \% \vee 20.5 \%$; $<0.05)$. Overall there were more dropouts with placebo than with all drugs (30.2\% with placebo $v 24.4 \%$ with drug treatment; significance not reported)

\section{CONCLUSIONS}

Pharmacotherapy is effective for the treatment of anxiety in generalised anxiety disorder compared with placebo. Benzodiazepines may be preferred to azapirones due to greater compliance, although there is no significant difference in efficacy between these drug classes.

For correspondence: Kristin Mitte, PhD Department of Psychology, University of Jena, Am Steiger 3, Hs 1, 07745 Jena, Germany; mail@kristin-mitte.de Source of funding: State Thuringia (Germany).

\section{NOTES}

There was evidence of publication bias in the trials included in the review. The main analysis used by the reviewers included effect sizes that had been inferred by the reviewers from significance levels in published studies. We have not presented the results from this analysis, as they are likely to underestimate true effect. We only present analyses of studies that computed effect sizes by means. Subgroup analyses, examining the efficacy of individual drugs, are not presented due to small sample sizes and lack of between drug comparisons.

\section{Commentary}

G eneralised anxiety disorder (GAD) was introduced as a residual category in DSM III. Diagnosis required duration of only four weeks. In DSM IIIR hierarchical restrictions were removed and duration was increased to six months. DSM IIIR also changed the character of anxiety complaints to focus on "unrealistic, excessive anxiety, and worry." DSM IV removed the "unrealistic" option, emphasised the experience of anxiety as being difficult to control, and kept the six month duration. ICD-10 is more flexible with duration requiring "several months".

The review by Mitte et al addresses treatment of GAD in studies from 1982 to 2002 . The earlier studies involve benzodiazepines (BZs; 34 studies) and azopirones (12 studies). Later studies include antidepressant drugs but only two studies of SSRIs are included in the review. All drugs were found to be superior to placebo with no differences between drug classes.

Current practice is to use SSRIs or SNRIs in GAD. Should the old practice of using BZs briefly in impairment causing short duration anxiety be reconsidered? Hesitation based on predictions of difficulty in getting patients to discontinue BZs will probably persist. Careful, planned management of BZ discontinuation after a few weeks when used in combination with other medications produced significant improvement in compliance and speed of recovery and all subjects discontinued. ${ }^{2}$ Does buspirone deserve a second look? The effects of buspirone on depressed mood in GAD were very positive. Most physicians will probably continue to prescribe antidepressants despite the delay in the onset of effect.

The review does have some limitations, which suggest caution if using the findings to support changing practice. Comparisons across drug groups are incomplete given the absence of studies after 2002, also the brevity of the early studies and the absence of standardised interviews in the majority of studies should be considered.

Richard P Swinson MD, FRCPC, FRCPsych McMaster University, Hamilton, Ontario, Canada

1 Kessler RC, Brandenburg N, Lane M, et al. Rethinking the duration requirement for generalized anxiety disorder: evidence from the National Comorbidity Survey Replication. Psychol Med 2005;35:1073-82.

2 Goddard AW, Brouette T, Almai A, et al. Early coadministration of clonazepam with sertraline for panic disorder. Arch Gen Psychiatry $2001 ; 58: 681-6$ 